\title{
Inoculación micorrícica de Pinus ponderosa en la Patagonia Argentina: colonización de las raíces, descripción de morfotipos y crecimiento de las plántulas en vivero
}

\author{
Mycorrhizal inoculation of Pinus ponderosa in Patagonia, Argentina: rootlets colonization, \\ morphotypes descriptions and seedling growth in nursery
}

\author{
Carolina Barroetaveña ${ }^{a, b *}$, Vilma N Bassani ${ }^{b}$, Mario Rajchenberg ${ }^{a, b}$

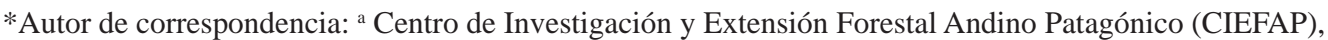 \\ Área Protección Forestal, C.C. 14 (9200) Esquel, Chubut, Argentina, tel.: 542945 453948, cbarroetavena@ciefap.org.ar \\ b Universidad Nacional de la Patagonia S.J. Bosco, Dpto. Ingeniería Forestal, Esquel, Chubut, Argentina.
}

\begin{abstract}
SUMMARY
Selection of ectomycorrhizal species to inoculate seedlings of exotic Pinaceae is a necessary step to ensure establishment, survival and growth in the forests. The use of spores as inoculum source has proven to be efficient, accessible and low-cost. In this paper we pursued the following objectives: a) to evaluate the effectiveness of inoculation with spores of Rhizopogon roseolus, Suillus luteus Hebeloma mesophaeum and Tricholoma muricatum on Pinus ponderosa seedlings, b) to determine the effect of mycorrhization on the morphometry of seedlings at planting time and c) to describe the mycorrhizal morphotypes of each individual association. We verified the success of mycorrhization with spores of $S$. luteus, $R$. roseolus and H. mesophaeum; the first two present the highest values of colonization, thus validating this technique of inoculation as successful for these species. Inoculation with T. muricatum was not successful, which excludes this species as a possible source of inoculum. The morphology of morphotypes developed by $S$. luteus and $R$. roseolus was constant, though very variable in $H$. mesophaeum. Morphometric parameters of inoculated seedlings did not differ from those of non-inoculated control, corroborating results from other parts of the world.
\end{abstract}

Key words: ectomycorrhizas, Rhizopogon roseolus, Suillus luteus, Hebeloma mesophaeum, Tricholoma muricatum.

\section{RESUMEN}

La selección de especies ectomicorrícicas para inocular plántulas de pináceas exóticas es un paso necesario para asegurar el establecimiento, supervivencia y crecimiento en las forestaciones. La utilización de esporas como fuente de inóculo ha sido probada como eficiente, accesible y de bajo costo. En este trabajo se desarrollaron los siguientes objetivos: a) evaluar la efectividad de la inoculación con esporas de las especies Rhizopogon roseolus, Suillus luteus, Hebeloma mesophaeum y Tricholoma muricatum en la micorrización de plántulas de Pinus ponderosa, b) determinar el efecto de la micorrización sobre la morfometría de las plantas al momento de llevarlas a plantación y c) describir los morfotipos micorrícicos de cada asociación particular. Se verificó el éxito de la micorrización con esporas de $S$. luteus, $R$. roseolus y $H$. mesophaeum, las dos primeras con los valores más altos de colonización, validando esta técnica de inoculación como exitosa para estas especies. La inoculación con T. muricatum no resultó exitosa, debiéndose descartar esta especie como posible fuente de inóculo. La morfología de los morfotipos desarrollados por $S$. luteus y R. roseolus fue constante, aunque con amplias variaciones en $H$. mesophaeum. Los valores morfométricos de las plantas no difirieron con las del testigo, corroborándose resultados de otras partes del mundo.

Palabras clave: ectomicorrizas, Rhizopogon roseolus, Suillus luteus, Hebeloma mesophaeum, Tricholoma muricatum.

\section{INTRODUCCIÓN}

Las coníferas, y en particular el género Pinus, necesitan establecer asociaciones simbióticas con hongos ectomicorrícicos para proveerse de nutrientes esenciales, principalmente nitrógeno, fósforo y agua (Smith y Read 2008). La superioridad de las plántulas micorrizadas frente a las no micorrizadas en términos de establecimiento, supervivencia y crecimiento en las forestaciones ha sido ampliamente demostrada (Duñabeitia et al. 2004). Estos beneficios, sin embargo, varían con las condiciones ambientales y con la asociación particular de las especies involucradas en la simbiosis mutualista. Pinus ponderosa Dougl. ex Laws. es la especie más ampliamente usada para forestar los pastizales del pedemonte Andinopatagónico de Argentina. Crece naturalmente al este del cordón montañoso de las Cascadas en el noroeste de Norteamérica, formando allí asociaciones simbióticas con al menos 157 hongos ectomicorrícicos (Barroetaveña et al. 2007). En Argentina, las plantaciones se iniciaron alrededor de 50 años atrás y existen actualmente 80.000 ha forestadas aproximadamente (CFI-FUNDFAEP 2009). La zona forestable con esta 
especie posee un régimen de precipitación mediterráneo, determinando que la mayoría de los sitios de plantación se encuentren en zonas expuestas a severo estrés hídrico, por lo que la optimización de la simbiosis micorrícica es de vital importancia. Estudios anteriores sobre los hongos ectomicorrícicos asociados a P. ponderosa en la Patagonia, detectaron 20 taxones en viveros y plantaciones (Barroetaveña y Rajchenberg 2003, Barroetaveña et al. 2005, 2007, 2010), todos ellos coincidentes con los taxones más frecuentemente encontrados en plantaciones de pino alrededor del mundo (Barroetaveña et al. 2007), sin reportes de especies nativas propias de los bosques de Nothofagus spp.

Las ectomicorrizas, asociación entre el micelio fúngico y las raíces finas, pueden caracterizarse morfológicamente como morfotipos micorrícicos (Agerer 1991), constituyendo una herramienta útil y económica para evaluar en vivero el estado de la simbiosis en las plantas.

En la Patagonia (Argentina), P. ponderosa es plantado mayoritariamente en pastizales anectotróficos, con flora nativa típicamente endomicorrícica (Fontenla et al. 2001). Por ello, los viveros constituyen la única instancia para agregar inóculo ectomicorrícico, aunque es necesario investigar aspectos relacionados a su incorporación exitosa en la producción de plántulas. Trabajos previos demostraron que los porcentajes de micorrización espontánea de los lotes de plantas producidas a raíz desnuda en la región variaron mucho entre viveros, con valores entre 26 - $50 \%$ de micorrización (Barroetaveña y Rajchenberg 2003). Es posible que las pérdidas durante el primer año de plantación (25 - $30 \%$ de plantas luego del primer año), especialmente en sitios con precipitación baja (300 - $500 \mathrm{~mm}$ ), se deba al menos en parte a una colonización ectomicorrícica insuficiente. Si bien en otros lugares del mundo existen experiencias de inoculación ectomicorrícica en especies del género Pinus (Castellano y Molina 1989, Parladé et al. 1996, Pera y Parladé 2005), existen pocos reportes sobre experiencias de inoculación y selección de especies para Pinus spp. en la Patagonia. Los trabajos de Peredo et al. (1992) y Martínez et al. (2007) en P. ponderosa y Chávez et al. (2009) en Pinus radiata D. Don (pino radiata) constituyen los únicos antecedente de este tipo de estudios en la región.

La incorporación de ectomicorrizas se puede realizar utilizando inóculo vegetativo (micelio); sin embargo, los viveros comerciales frecuentemente utilizan esporas como fuente de inóculo (Rincón et al. 2001, Steinfeld et al. 2003), debido a que estas no necesitan de una fase de cultivo puro como el inóculo vegetativo, ocupan poco volumen, pueden tolerar períodos de almacenamiento largos, en general son muy abundantes en los esporocarpos y su aplicación es sencilla, ya que se las puede agregar en el agua de riego (Parladé et al. 1996, Rincón et al. 2001). La selección de las especies micorrícicas a utilizar debe considerar la eficacia, la disponibilidad y la logística de obtención del inóculo. Rhizopogon roseolus (Corda) Th. Fr. (Rhizopogonaceae, Boletales, Basidiomycota), Suillus luteus (L.) Roussel (Suillaceae, Boletales, Basidiomycota) y Hebeloma mesophaeum (Pers.) Quél. (Strophariaceae, Agaricales, Basidiomycota) son especies que fructifican de manera abundante en plantaciones de la región patagónica de argentina, constituyendo buenas fuentes de inóculo esporal y fueron seleccionadas como candidatas para su empleo en viveros (Barroetaveña et al. 2005). Tricholoma muricatum Shanks (Tricholomataceae, Agaricales, Basidiomycota), de fructificación muy abundante en plantaciones con suelos húmedos cercanos a mallines o cursos de agua de la región Patagónica de Argentina, también aparece como una opción interesante.

Bajo las hipótesis de que las fructificaciones de especies ectomicorrícicas presentes en las plantaciones de P. ponderosa en la Patagonia constituyen una fuente de inóculo efectivo para las plántulas de vivero, que las diferentes especies de hongos ectomicorrícicos pueden establecer proporciones diferentes de micorrizas con las raíces finas de las plántulas, y que la simbiosis con diferentes especie ectomicorrícicas puede impactar positiva o negativamente los parámetros morfométricos de las plántulas en vivero, se plantean en este trabajo los siguientes objetivos: a) evaluar la efectividad de la inoculación con esporas de Rhizopogon roseolus, Suillus luteus, Hebeloma mesophaeum y Tricholoma muricatum en la micorrización de plántulas de $P$. ponderosa, b) determinar el efecto de la micorrización sobre la morfometría de las plantas al momento de llevarlas a plantación, y c) describir los morfotipos micorrícicos de cada asociación particular.

\section{MÉTODOS}

Producción de las plántulas. El estudio se realizó con plántulas provenientes del vivero de producción acelerada de plántulas forestales de la Universidad Nacional de la Patagonia S. J. Bosco, Esquel, Chubut, Argentina. Se utilizaron plántulas de 10 meses, una vez concluidas las fases de fertilización, a fin de emular una situación productiva y considerando los efectos inhibitorios de la fertilización sobre la micorrización (Castellano y Molina 1989). La siembra se efectuó durante la primer semana de agosto de 2003 con semillas de calidad comercial provenientes de EEA INTA Trevelin (Chubut, Argentina) sobre sustrato inerte ( $50 \%$ turba $+50 \%$ arena volcánica). Se aplicó fertirriego desde fin de agosto de 2003 hasta mediados de mayo de 2004 (tres veces por semana entre agosto - diciembre, y cada tres días entre diciembre - mayo), con dosis nitrógeno - fósforo - potasio de 55 - 167 - 64,5 $\mathrm{mg} \mathrm{L}^{-1}$ en la fase de establecimiento, 118 - 30 - $37 \mathrm{mg} \mathrm{L}^{-1}$ en la fase de crecimiento rápido y 19,5 - 69 - $14 \mathrm{mg} \mathrm{L}^{-1}$ en la fase de endurecimiento. La temperatura media del interior del invernáculo fue de $17^{\circ} \mathrm{C}$ con extremos de 5 y $25^{\circ} \mathrm{C}$.

Repique. Las plántulas seleccionadas se trasplantaron a macetas plásticas de $550 \mathrm{~cm}^{3}$, agregando una mezcla 2:1 de tierra negra tindalizada en vapor a $100{ }^{\circ} \mathrm{C}$ por 60 minutos, tres veces a intervalo de una semana (Torres y Hon- 
rrubia 1994b) y arena volcánica esterilizada en estufa a $130{ }^{\circ} \mathrm{C}$ durante 12 horas.

Inoculación micorrícica. Se aplicaron cuatro tratamientos utilizando suspensiones de esporas de Suillus luteus, $\mathrm{He}$ beloma mesophaeum, Rhizopogon roseolus y Tricholoma muricatum, además de un testigo sin inocular.

Las suspensiones de esporas se prepararon a partir de cuerpos fructíferos colectados en plantaciones aledañas a la ciudad de Esquel (Argentina) durante mayo de 2004. Inmediatamente en laboratorio se trituraron ejemplares de cada especie con agua destilada estéril y se guardaron en frascos cerrados a $4{ }^{\circ} \mathrm{C}$ hasta ser usadas. Se determinó la concentración de esporas de cada suspensión usando una cámara de conteo Neubauer.

La inoculación se llevó a cabo inmediatamente luego del trasplante a macetas en junio de 2004, aplicando una dosis de $2 \times 10^{7}$ esporas de $H$. mesophaeum, y 6 × $10^{7}$ esporas de T. muricatum, $R$. roseolus y $S$. luteus por planta (Pera y Parladé 2005), consideradas equivalentes para el análisis estadístico. Para ello se efectuó un pequeño hoyo en la tierra, cerca del tallo de cada planta, y se aplicó la dosis de esporas usando una jeringa diferente para cada tratamiento. Las plantas se mantuvieron nueve meses en invernáculo y se regaron a demanda únicamente con agua proveniente de una perforación.

Evaluación de la micorrización y del crecimiento. Del total de lotes de plántulas inoculadas, se seleccionaron 10 de cada tratamiento para efectuar el seguimiento en vivero del efecto de las inoculaciones en la micorrización y en el crecimiento. Para ello se evaluó la morfometría de esas plántulas antes de realizar las inoculaciones y tras nueve meses, momento en que también se cuantificó la micorrización. Antes de aplicar las inoculaciones se evaluó la micorrización en otras 10 plantas sacrificadas para tal fin al momento del trasplante.

Respecto a la morfometría, se evaluaron el diámetro del cuello y el largo del vástago (desde el cuello hasta el brote apical). La micorrización se evaluó en cinco raíces laterales elegidas al azar (entre 88 y $521 \mathrm{~cm}$ de largo total) por planta. Para ello se observaban, en una lupa binocular con 6,5 - 25 aumentos, las raíces sumergidas en agua, contando el número de puntas de raíces micorrizadas y no micorrizadas, a la vez que se iban clasificando los morfotipos.

Descripción de los morfotipos. Se separaron bajo la lupa puntas micorrícicas bien desarrolladas de cada morfotipo. Sobre la base de la metodología de Agerer (1991) se describieron el tipo de ramificación, el largo, el color y el aspecto de la superficie externa, la presencia de rizomorfos o de hifas emanantes. Se realizaron preparados microscópicos en agua para evaluar la estructura del manto, la forma y dimensiones de las hifas, presencia y tipo de septos y concreciones de cristales sobre las paredes u ornamentaciones.
Análisis estadístico. Las diferencias entre la medias de micorrización y las variables morfométricas se evaluaron mediante análisis de varianza de un factor (ANDEVA) y comparaciones múltiples (prueba de Tukey) o prueba de Welch y comparaciones múltiples (pueba de GamesHowell) cuando las varianzas fueron desiguales. En todos los casos se utilizó un nivel de significación del $95 \%$, la normalidad de los datos se determinó mediante la prueba de Shapiro-Wilk y la igualdad de varianzas utilizando la prueba de Levene. Se utilizó el paquete estadístico SPSS versión 11.5 para Windows.

\section{RESULTADOS}

Micorrización. El análisis de las plántulas antes de la inoculación arrojó valores nulos de micorrización. A los nueve meses de la inoculación, todos los tratamientos mostraron micorrización salvo el inoculado con T. muricatum, que fue eliminado de los análisis. El porcentaje de micorrización mostró diferencias significativas entre tratamientos (prueba de Welch, $P=0,01$ ), siendo $S$. $l u$ teus el tratamiento con valor más alto (media $=38,4 \%$ ), significativamente mayor que $H$. mesophaeum (media = $11,4 \%$ ) y el testigo (media $=9,6 \%$ ) (prueba GamesHowell), seguido por $R$. roseolus (cuadro 1). Se detectaron contaminaciones en todos los tratamientos (cuadro 1), con porcentajes inferiores al $5 \%$.

Morfometría. Los valores iniciales del largo del vástago no mostraron diferencias significativas entre los grupos variando entre 9,2 - 10,4 cm (ANDEVA, $P=0,47$ ). A los nueve meses las diferencias entre mediciones no fueron significativas (ANDEVA, $P=0,57$ ), como así tampoco el largo final del vástago (prueba de Welch, $P=0,32$ ), aunque en ambos casos se observó que el tratamiento $S$. luteus presentaba un valor mayor (cuadro 1). El diámetro del cuello no mostró diferencias significativas en la medición inicial, variando entre 4,3 - $5 \mathrm{~mm}$ (ANDEVA, $P=0,29$ ), y tampoco antes de la plantación (ANDEVA, $P=0,56$ ) (cuadro 1).

Descripción del morfotipo Rhizopogon roseolus - P. ponderosa. Puntas dicotómicas, con ramas cortas, simples, múltiples (coraloides) o formando cabezuelas, color blanco, ocasionalmente con zonas rosáceas, textura afieltrada, sin brillo o ligeramente satinado, con ápices blancos a amarillentos. El sistema de 2,2 - 6,5 mm de largo $\times 1,5-$ $7,8 \mathrm{~mm}$ de ancho y cada punta 1,8 - 2,2 mm de largo $\times 0,35$ $-0,45 \mathrm{~mm}$ de diámetro, con forma recta. No se ve a través del manto, que cubre homogéneamente la raíz (figura 1A). Sin hifas emanantes o muy escasas, con septos simples. Rizomorfos abundantes, ramificados en ángulos agudos, color blanco, ligeramente plumosos con los bordes lisos, de $20-80 \mu \mathrm{m}$ de diámetro, con muchos cristales hialinos adheridos, anatómicamente ligeramente diferenciados, compactos, con hifas centrales más gruesas que las demás, con septos disueltos. Manto externo plectenquimatoso, a 
Cuadro 1. Porcentaje de micorrización, parámetros morfométricos y especies contaminantes en plántulas inoculadas y testigo al momento de la plantación.

Percentage of mycorrhization, morphometric parameters and contaminant species in inoculated and control seedlings at time of planting.

\begin{tabular}{lcccl}
\hline \multicolumn{1}{c}{ Tratamiento } & Micorrización* $(\%)$ & Largo vástago $(\mathrm{cm})$ & Diámetro cuello $(\mathrm{mm})$ & \multicolumn{1}{c}{ Contaminantes } \\
\hline Suillus luteus & $38,4(5,89) \mathrm{a}$ & $25,0(1,21)$ a & $5,3(0,21)$ a & Cenococcum sp. \\
Hebeloma mesophaeum & $11,4(2,38) \mathrm{b}$ & $23,4(1,83)$ a & $5,2(0,24)$ a & E-strain \\
Rhizopogon roseolus & $34,9(7,42)$ ab & $21,9(1,02)$ a & $5,0(0,21)$ a & E-strain; Cenococcum sp. \\
Testigo & $9,64(3,36) \mathrm{b}$ & $23,4(0,89)$ a & $5,5(0,19)$ a & Cenococum sp.; Rhizopogon sp. \\
\hline
\end{tabular}

Diferentes letras significan diferencias significativas con la prueba de Welch o prueba de Games-Howell, $P<0,05$. Entre paréntesis el error estándar de la media. *Sin incluir contaminantes.

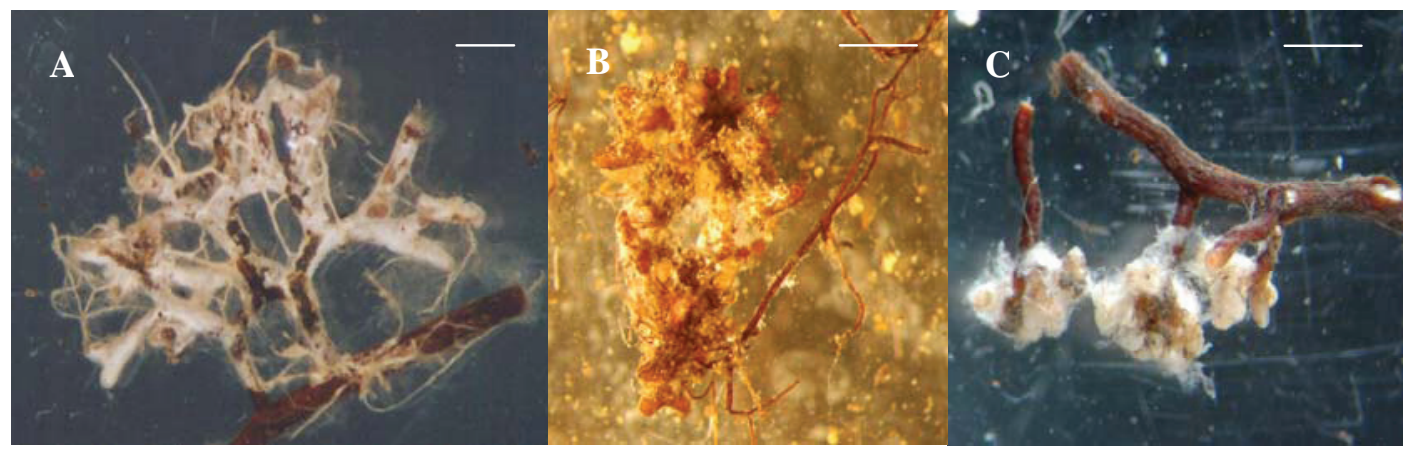

Figura 1. Aspecto general de los morfotipos micorrícicos formados por (A) Rhizopogon roseolus, (B) Suillus luteus y (C) Hebeloma mesophaeum con Pinus ponderosa. La barra equivale a $1 \mathrm{~mm}$.

General appearance of ectomycorrhizal morphotypes formed by (A) Rhizopogon roseolus, (B) Suillus luteus and (C) Hebeloma mesophaeum with Pinus ponderosa. The bar is equal to $1 \mathrm{~mm}$.

veces con disposición circular difícil de observar debido a la presencia de abundantes cristales hialinos adheridos a las paredes de las hifas, que tienen septos simples y de 2 $3 \mu \mathrm{m}$ de diámetro. Manto interno plectenquimatoso, apretado y con disposición irregular, hifas con septos simples, sin cristales sobre las paredes, de 3 - $4 \mu \mathrm{m}$ de diámetro.

Descripción del morfotipo Suillus luteus - P. ponderosa. Puntas dicotómicas simples o múltiples, color castaño claro con parches blanquecinos ligeramente nacarados, textura lanosa, brillo nulo a ligeramente satinado fuera de los parches, ápices amarillentos (figura 1B). El sistema de la micorriza tiene 1,6 - 3,2 mm de largo $\times 1,8-3,2 \mathrm{~mm}$ de ancho, con puntas individuales de 0,7 - 1,3 mm de largo $\times 0,2-0,3 \mathrm{~mm}$ de diámetro, rectas o con constricciones regulares. Se ve a través del manto. Hifas emanantes abundantes, alargadas, de $4 \mu \mathrm{m}$ de diámetro, septos simples y paredes ligeramente engrosadas con cristales poliédricos con bordes redondeados de tamaños diversos. Rizomorfos muy frecuentes, color pardo oscuro, ramificados, de 150 - $160 \mu \mathrm{m}$ de diámetro, estructura indiferenciada, con hifas alargadas de 1,5 - $3 \mu \mathrm{m}$ de diámetro, paredes delgadas lisas, ocasionalmente con cristales poliédricos con bordes redondeados de tamaños diversos. Manto externo plectenquimatoso, con hifas alar- gadas, castañas, de 2,5 - $3 \mu \mathrm{m}$ de diámetro, holgadamente entrelazadas dispuestas sin patrón específico. Manto interno pseudoparenquimatoso, con hifas ramificadas, de forma irregular, entrelazadas, de 2 - 4 de diámetro, paredes ligeramente engrosadas y septos simples.

Descripción del morfotipo Hebeloma mesophaeum - P. ponderosa. Puntas dicotómicas simples o múltiples, color ocre amarillentas con manto traslúcido con parches blancos ocasionales, textura afieltrada, sin brillo salvo los parches blancos nacarados, y ápices traslúcidos (figura $1 \mathrm{C}$ ). El sistema de 2 - $3 \mathrm{~mm}$ de largo $\times 2$ - 4,6 mm de ancho y cada punta 0,3 $-0,4 \mathrm{~mm}$ de diámetro, derechas o curvadas. Se ve a través del manto donde no hay parches. Hifas emanantes abundantes, aspecto lanoso, crémeo-amarillentas, fibuladas, hialinas, con paredes levemente engrosadas, de 3 - 4,5 $\mu \mathrm{m}$ de diámetro, ocasionalmente con depósitos amorfos sobre las paredes. Sin rizomorfos. Manto externo plectenquimatoso con hifas dispuestas irregularmente sin patrón discernible, de $3-4,5 \mu \mathrm{m}$ de diámetro, fibuladas, con contenidos ocre-amarillentos y paredes lisas, ligeramente engrosadas. Manto interno transicional entre plectenquimatoso y pseudoparenquimatoso, con hifas de forma irregular, apretadamente entrelazadas, de 2 - 6 $\mu \mathrm{m}$ de ancho, hialinas a amarillentas, con paredes engrosadas. 


\section{DISCUSIÓN}

La inoculación de las plántulas de $P$. ponderosa con esporas de los hongos ectomicorrícicos seleccionados es exitosa, salvo el caso de T. muricatum. Suillus luteus y $R$. roseolus son las especies que forman mayor cantidad de micorrrizas, aunque ello no se traduce en un crecimiento significativamente mayor.

Las especies que colonizan exitosamente las raíces de P. ponderosa han sido reportadas como pioneras (earlysatage) en el caso de Hebeloma sp. (Marmeisse et al. 1999), o como multi-etapa en los casos de Rhizopogon sp. y Suillus sp.(Dahlberg y Finlay 1999, Molina et al. 1999), características que se han considerado como propicias para la inoculación de plántulas. Ambos géneros aparecen asociados a diferentes especies del género Pinus.

Suillus luteus se ha utilizado en experimentos de inoculación a gran escala en diferentes continentes. Castellano y Molina (1989) reportan inoculaciones exitosas para 12 combinaciones de especies del género, en general con respuestas no significativas en el crecimiento. Torres y Honrubia (1994a) y González Ochoa et al. (2003) reportaron inoculaciones exitosas en especies de Pinus europeas, en este último caso con mejor respuesta de las esporas que el micelio. En la Patagonia (Argentina) la especie se encuentra ampliamente distribuida en plantaciones de Pinus spp. bajo diferentes condiciones ambientales fructificando abundantemente y presentando alta concentración de esporas por gramo de fructificación fresca $\left(1,01 \times 10^{7}\right)$ (Barroetaveña et al. 2005), asegurando la provisión de esporas para este tipo de inoculaciones.

Rhizopogon roseolus ofrece numerosas ventajas comparativas frente a otras especies ectomicorrícicas para el género Pinus, pues logra una buena colonización de los sistemas radicales de plántulas producidos en maceta y ferti-irrigadas (Rincón et al. 2001, Martínez et al. 2007), posee alta tolerancia a las condiciones medioambientales adversas (Duñabeitia et al. 2004) y presenta numerosas hifas y rizomorfos que penetran en el suelo maximizando la captura de agua nutrientes (Molina et al. 1999). Además, fructifica abundantemente presentando altas concentraciones de esporas por cuerpo fructífero (56,15 x $10^{7}$ esporas por gramo de fructificación fresca); sus esporas poseen una alta tasa de germinación, una gran capacidad de colonizar las raíces con dosis bajas y de mantener su viabilidad luego del almacenamiento a bajas temperaturas (Molina y Trappe 1994, Torres y Honrubia 1994a). En España ha sido utilizado en estudios de inoculación, crecimiento y supervivencia sobre Pinus pinea L. (Rincón et al. 2001), Pinus halepensis Mill. (Torres y Honrubia 1994b), Pinus pinaster Ait. y Pseudotsuga menziesii (Mirb.) Franco (Parladé et al. 1996).

Diferentes cepas del género Hebeloma demuestran mejorar el crecimiento de los árboles tanto en condiciones de vivero como en plantación y son utilizadas como inóculo comercial (Marmeisse et al. 1999). Tanto las inoculacio- nes con esporas como con micelio son reportadas como exitosas, especialmente la especie $H$. crustuliniforme (Marmeisse et al. 1999). En este estudio, sin embargo, la colonización con H. mesophaeum fue baja. Puede contribuir a este resultado la menor dosis de esporas aplicada, debido a la menor disponibilidad de esporas al momento de inocular originada por la muy baja concentración de esporas en sus cuerpos fructíferos $\left(1,9 \times 10^{6}\right.$ esporas por gramo de fructificación fresca). Sin embargo, la magnitud de la dosis aplicada fue superior a las dosis recomendadas por trabajos precedentes $\left(10^{5}-10^{6}\right.$ esporas por planta) para inoculaciones en Pinus spp. (Pera y Parladé 2005), que reportan que dosis mayores no incrementan la micorrización (Parladé et al. 1996, Rincón et al. 2001). La baja viabilidad de las esporas en fructificaciones frescas, la baja tasa de germinación y la rápida pérdida de viabilidad con el almacenamiento encontrados en este género (Torres y Honrubia 1994, Marmeisse et al. 1999) están posiblemente más vinculadas a dicho resultado.

Si bien existen antecedentes sobre inoculaciones exitosas con micelio de Tricholoma spp. (Duñabeitia et al. 1996), no se encontraron antecedentes de inoculaciones con esporas. El género ha sido reportado como de estado sucesional avanzado (late-stage) (Smith y Read 2008) y la germinación de sus basidiosporas como lenta e irregular en medios de cultivo simples (Fries 1984). Torres y Honrubia (1994a) reportan pérdida drástica y rápida de viabilidad en las esporas de T. terreum. Las dificultades para germinar, sumado a la drástica pérdida de viabilidad pueden explicar la nula micorrización con esta especie.

La ausencia de diferencias morfométricas entre las plantas micorrizadas y el testigo no es relevante en la fase de vivero ya que las plantas son provistas con todos los nutrientes y el agua que necesitan, por lo cual los beneficios de la micorrización no se manifiestan. Puede ocurrir que las plantas micorrizadas sean incluso más pequeñas que las no micorrizadas, pues tienen un gasto energético mayor destinado a mantener la simbiosis (Smith y Read 2008) o bien que factores como el tipo de sustrato, los niveles de nutrientes presentes en él o el tamaño de los contenedores influencien negativamente el estímulo en el crecimiento (Rincón et al. 2001). Los incrementos en prendimiento y crecimiento que se detectan en la plantación, donde las condiciones ambientales cambian abruptamente y para lo cual la planta con micorrizas se encuentra mucho mejor preparada, son los parámetros a tener en cuenta al momento de decidir realizar inoculaciones.

Se detectaron contaminaciones con otros hongos ectomicorrícicos al momento de evaluar las raíces antes de la plantación, sin embargo no se había detectado colonización previa a la inoculación. Esto puede deberse a que la evaluación inicial se realizó cuando las plántulas venían de recibir alta dosis de fertirriego, que es sabido inhibe el establecimiento de micorrizas (Smith y Read 2008). Posteriormente, ya sin fertirriego, las plántulas pudieron desarrollar asociaciones con inóculo pre-existente o que 
pudiera haber llegado por aire. Especialmente entre ellas se destacaron los morfotipos "E-strain" (puntas simples o dicotómicas, rectas o curvadas, amarillentas a pardas sin brillo, sin hifas emanantes ni rizomorfos, con manto muy delgado), originados por Ascomycetes pertenecientes al género Wilcoxina Chin S. Yang et Korf (Barroetaveña et al. 2010), y morfotipos correspondientes al género Cenococcum Moug. et Fr. (puntas simples, rectas, negras con brillo satinado, con hifas emanantes negras, gruesas y abundantes), ambas con características pioneras, de carácter cosmopolita, aunque no buenas competidoras frente a otras especies. Si bien su abundancia fue baja en todos los tratamientos, incluso en el testigo sin inocular, donde no tenían competencia por las puntas de raíces, su abundancia puede crecer en situaciones donde hay un aporte continuo de inóculo, moderada o baja fertilización y ausencia de otras especies ectomicorrícicas.

La descripción del morfotipo Rhizopogon roseolus + Pinus ponderosa coincidió con la de DEMMY (Agerer y Rambold 2010) y Torres et al. (1991), salvo en el color marrón claro descrito para los morfotipos maduros. Sus caracteres diagnósticos son la ramificación dicotómica, a veces en clusters, el manto afieltrado, plectenquimatoso, blanco a rosáceo, cubierto por cristales traslúcidos muy abundantes, hifas emanantes con septos simples y abundantes rizomorfos blancos. El morfotipo Suillus luteus + Pinus ponderosa coincidió en el color, el patrón de ramificación y la presencia de hifas emanantes y rizomorfos con la descripción de Riffle (1973) para la asociación Suillus granulatus + Pinus ponderosa y con otras asociaciones Pinus + Suillus descritas por otros autores (Torres et al. 1991, Nourha y Becerra 2001). Sus caracteres diagnósticos son la ramificación dicotómica simple o múltiple, el manto castaño claro con parches blanquecinos ligeramente nacarados, abundantes hifas emanantes con septos simples con cristales poliédricos sobre las paredes, rizomorfos muy frecuentes, ramificados y de color pardo oscuro. El morfotipo Hebeloma mesophaeum + Pinus ponderosa coincidió parcialmente con la descripción de DEEMY (Agerer y Rambold 2010). Como ya reportaron Marmeisse et al. (1999) y Barroetaveña et al. (2010), las especies del género presentan amplias variaciones morfológicas, que complican su determinación empleando solamente la morfología y la anatomía del morfotipo. Sus caracteres diagnósticos básicos son la ramificación dicotómica simple o múltiple, el manto completo o en parches afieltrado, brillante, blanco a amarillento, y con abundantes hifas emanantes fibuladas.

\section{CONCLUSIONES}

Se verifica el éxito de la micorrización de plántulas de $P$. ponderosa crecidas en invernáculo con esporas de los hongos ectomicorrícicos Suillus luteus, Rhizopogon roseolus y Hebeloma mesophaeum, que constituyen fuentes de inóculo accesibles material y económicamente para los vi- veros del sur de Patagonia dada su fructificación conspicua y abundante. Se observa constancia en la morfología de los morfotipos desarrollados en cada asociación, aunque con amplias variaciones en el caso de H. mesophaeum, lo cual permite evaluar el establecimiento de cada una de estas simbiosis con técnicas sencillas de microscopia óptica. Los niveles de contaminación con otras especies micorrícicas es muy bajo o nulo en los tratamientos inoculados, mostrando que las especies probadas compiten ventajosamente con ellos. La inoculación con T. muricatum no resulta exitosa, debiéndose descartar esta especie como posible fuente de inóculo esporal. Los valores morfométricos de las plantas no difieren con las del testigo, corroborándose resultados de otras partes del mundo. Si bien $R$. roseolus y $S$. luteus son las especies con valores más altos de colonización, la eficiencia de las diferentes especies en la supervivencia y crecimiento de las plántulas en plantación debe ser evaluada.

\section{AGRADECIMIENTOS}

$\mathrm{Al}$ vivero del PAIDER forestal de la Universidad Nacional de la Patagonia S.J. Bosco sede Esquel, y especialmente al Sr. Eduardo Núñez por las plántulas usadas en este ensayo y su colaboración con información y sugerencias durante la ejecución del trabajo. Al Téc. For. Juan Monges por su colaboración en tareas de campo y a la Dra. Ludmila La Manna por las sugerencias durante la escritura del manuscrito. Este trabajo fue financiado la Agencia Nacional de Promoción Científica y Tecnológica de Argentina, FONCYT- PICT 8674/00, proyecto: "Micorrizas e implantación de Pinus ponderosa en Patagonia: diversidad, eficiencia y calidad de la micorrización". CB y MR son miembros de la carrera del investigador científico del CONICET.

\section{REFERENCIAS}

Agerer R. 1991. Characterization of Ectomycorrhizae. In Norris JR, DJ Read, AK Varma eds. Techniques for the study of mycorrhiza, Methods in microbiology, vol. 23. Londres. Academic Press. p. 25-73.

Agerer R, G Rambold. 2004-2010. DEEMY, An Information System for Characterization and Determination of Ectomycorrhizae. Canadian Journal of Forest Research 40(10): 1940-1950. DOI: 10.1139/X10-135.

Barroetaveña C, M Rajchenberg. 2003. Las micorrizas y la producción de plántulas de Pinus ponderosa Dougl. et Laws. en la Patagonia Argentina. Bosque 24(1): 17-33.

Barroetaveña C, M Rajchenberg, E Cázares. 2005. Mycorrhizal fungi in Pinus ponderosa introduced in Central Patagonia (Argentina). Nova Hedwigia 80: 453-464.

Barroetaveña C, E Cazares M Rajchenberg. 2007. Ectomycorrhizal fungal species associated with Ponderosa pine and Douglas fir: a comparison of species richness in native forests and Patagonian plantations. Mycorrhiza 17: 355-373.

Barroetaveña C, MB Pildain, ME Salgado Salomon, JL Eberhart. 
2010. Molecular identification of ectomycorrhizas associated with ponderosa pine seedlings in Patagonian nurseries (Argentina). Canadian Journal of Forest Research 40: 1940-1950. DOI: 10.1139/X10-135.

Castellano M, R Molina. 1989. Mycorrhizae. In Landis Tinus TR, S Mc Donald, J Barnett eds. The Container Tree Nursery Manual vol. 5. Washington DC. U.S. Department of Agriculture -Forest Service. p. 101-167. (Agricultural Handbook 674).

CFI-FUNDFAEP (Consejo Federal de Inversiones-Fundación para el desarrollo forestal, ambiental y del ecoturismo Patagónico, AR). 2009. Inventario del bosque implantado de la provincia del Neuquén. Buenos Aires, Argentina. Consejo Federal de Inversiones. 109 p.

Chávez DM, CG Pereira. A Machuca. 2009. Efecto de tipos de inóculos de tres especies fúngicas en la micorrización controlada de plántulas de Pinus radiata. Bosque 30(1): 4-9.

Dahlberg A, RD Finlay. 1999. Suillus. In Cairney JWG, SM Chambers eds. Ectomycorrhizal fungi: key genera in profile. Berlin, Alemania. Springer. p 33-64.

Duñabeitia MK, S Hormilla, I Salcedo, JI Peña. 1996. Ectomycorrhizae synthesized between Pinus radiata and eight fungi associated with Pinus spp. Mycologia 88: 897-908.

Duñabeitia MK, S Hormilla, J Garcia-Plazaola, K Txarterina, U Arteche, JM Becerril. 2004. Differential responses of three fungal species to environmental factors and their role in the mycorrhization of Pinus radiata D. Don. Mycorrhiza 14: 11-18.

Fontenla S, O Puntieri, JA Ocampo. 2001. Mycorrhizal associations in the Patagonian steppe, Argentine. Plant and Soil 232: 13-29.

Fries N. 1984. Spore germination in the higher Basidiomycetes. Proceedings of the Indian Academy of Science 93 (3): 205222.

González-Ochoa AI, J de las Heras, P Torres, E Sánchez-Gómez. 2003. Mycorrhization of Pinus halepensis Mill. and Pinus pinaster Aiton seedlings in two commercial nurseries. Annals of Forest Science 60: 43-48.

Marmeisse R, H Gryta, P Jargeat, L Fraissinet-Tacher, G Gray, JC Debaud. 1999. Hebeloma. In Cairney JWG, SM Chambers eds. Ectomycorrhizal fungi: key genera in profile. Berlin, Alemania. Springer. p. 89-128.

Martínez DB, C Barroetaveña, M Rajchenberg. 2007. Influencia del régimen de fertilización y del momento de inoculación en la micorrización de Pinus ponderosa en la etapa de vive- ro. Bosque 28(3): 226-233.

Molina R, JM Trappe. 1994. Biology of the ectomycorrhizal genus Rhizopogon I: Host associations, host specificity and pure culture syntheses. New Phytologist 126: 653-675.

Molina R., JM Trappe, LC Grubisha, JW Spatafora. 1999. Rhizopogon. In Cairney JWG, SM Chambers eds. Ectomycorrhizal fungi: key genera in profile. Berlin, Alemania. Springer. p 129-186.

Nourha ER, A Becerra. 2001. Síntesis micorrícica de Suillus granulatus (Eumycota) y plantines de Pinus elliottii (Pinaceae). Boletín de la Sociedad Argentina de Botánica 36: 209-215.

Parladé J, J Pera, IF Alvarez. 1996. Inoculation of containerized Pseudotsuga menziesii and Pinus pinaster seedlings with spores of five species of ectomycorrhizal fungi. Mycorrhiza 6: $237-245$.

Pera J, J Parladé. 2005. Inoculación controlada con hongos ectomicorrícicos en la producción de planta destinada a repoblaciones forestales: estado actual en España. Investigación Agraria, Sistema y Recursos Forestales 14(3): 419-433.

Peredo H, O Alonso, E Valenzuela. 1992. Inoculación micorrícica de Pinus ponderosa en el vivero forestal Junín de los Andes, Argentina. Ciencia e Investigación Forestal 6: 157-167.

Riffle JW. 1973. Pure culture synthesys of ectomycorrhizae on Pinus ponderosa with species of Amanita, Suillus and Lactarius. Forest Science 19: 242-250.

Rincón AM, IF Alvarez, J Pera. 2001. Inoculation of containerized Pinus pinea L. seedlings with seven ectomycorrhizal fungi. Mycorrhiza 11(6): 265-271.

Smith SE, DJ Read. 2008. Mycorrhizal symbiosis $3^{\text {th }}$ ed. Cambridge, Inglaterra. Academic Press. 605 p.

Steinfeld D, MP Amaranthus, E Cazares. 2003. Survival of ponderosa pine (Pinus ponderosa Dougl. ex Laws.) seedlings outplanted with Rhizopogon mycorrhizae inoculated with spores at the nursery. Journal of Arboriculture 29: 197-207.

Torres P, M Honrubia, MA Morte. 1991. In vitro síntesis of ectomycorrhizae between Suillus collinitus (Fr.) O. Kuntze and Rhizopogon roseolus (Corda) Th. M. Fr. with Pinus halepensis Miller. Mycotaxon 41: 437-443.

Torres P, M Honrubia. 1994a. Basidiospores viability in stored slurries. Mycological Research 98(5): 527-530.

Torres P, M Honrubia. 1994b. Inoculation of containerized Pinus halepensis seedlings with basidiospores of Pisolithus tinctorius, Rhizopogon roseolus and Suillus collinitus. Annales des Sciences Forestieres 51(5): 521-528. 
